\title{
BMJ Open Impulsivity-focused group intervention to reduce binge eating episodes in patients with binge eating disorder: study protocol of the randomised controlled IMPULS trial
}

\author{
Kathrin Schag, ${ }^{1}$ Elisabeth J Leehr, ${ }^{1}$ Peter Martus, ${ }^{2}$ Wolfgang Bethge ${ }^{3}$ \\ Sandra Becker, ${ }^{1}$ Stephan Zipfel, ${ }^{1}$ Katrin E Giel ${ }^{1}$
}

To cite: Schag K, Leehr EJ, Martus $\mathrm{P}$, et al. Impulsivityfocused group intervention to reduce binge eating episodes in patients with binge eating disorder: study protocol of the randomised controlled IMPULS trial. BMJ Open 2015;5:e009445.

doi:10.1136/bmjopen-2015009445

- Prepublication history for this paper is available online. To view these files please visit the journal online (http://dx.doi.org/10.1136/ bmjopen-2015-009445).

Received 19 July 2015 Revised 13 October 2015 Accepted 14 October 2015

\section{CrossMark}

\footnotetext{
${ }^{1}$ Medical University Hospital Tübingen, Department of Psychosomatic Medicine and Psychotherapy, Tübingen, Germany

${ }^{2}$ Medical University Hospital Tübingen, Institute for Clinical Epidemiology and Applied Biometry, Tübingen, Germany

${ }^{3}$ Medical University Hospital Tübingen, Center for Clinical Studies, Tübingen, Germany
}

Correspondence to Dr Kathrin Schag; kathrin.schag@med. uni-tuebingen.de

\begin{abstract}
Introduction: The core symptom of binge eating disorder (BED) is recurrent binge eating that is accompanied by a sense of loss of control. BED is frequently associated with obesity, one of the main public health challenges today. Experimental studies deliver evidence that general trait impulsivity and disorder-specific food-related impulsivity constitute risk factors for BED. Cognitive-behavioural treatment (CBT) is deemed to be the most effective intervention concerning BED. We developed a group intervention based on CBT and especially focusing on impulsivity. We hypothesise that such an impulsivity-focused group intervention is able to increase control over impulsive eating behaviour, that is, reduce binge eating episodes, further eating pathology and impulsivity. Body weight might also be influenced in the long term.
\end{abstract}

Methods and analysis: The present randomised controlled trial investigates the feasibility, acceptance and efficacy of this impulsivity-focused group intervention in patients with BED. We compare 39 patients with BED in the experimental group to 39 patients with BED in the control group at three appointments: before and after the group intervention and in a 3-month follow-up. Patients with BED in the experimental group receive 8 weekly sessions of the impulsivity-focused group intervention with 5-6 patients per group. Patients with BED in the control group receive no group intervention. The primary outcome is the binge eating frequency over the past 4 weeks. Secondary outcomes comprise further eating pathology, general impulsivity and food-related impulsivity assessed by eye tracking methodology, and body weight. Additionally, we assess binge eating and other impulsive behaviour weekly in process analyses during the time period of the group intervention.

Ethics and dissemination: This study has been approved by the ethics committee of the medical faculty of Eberhard Karls University Tübingen and the University Hospital Tübingen. Data are monitored by the Centre of Clinical Studies, University Hospital Tübingen.

\section{Strengths and limitations of this study}

- This clinical trial IMPULS will deliver evidence concerning food-related impulsivity as one potential treatment factor in binge eating disorder (BED). The findings of IMPULS will clarify the role of impulsivity in cognitive-behavioural interventions and might strengthen the implementation of self-control strategies and food-related cue exposure in BED treatment. Further, IMPULS explores the association between BED and addictive disorders.

- IMPULS is a randomised controlled trial with an experimental group receiving an impulsivity focused group intervention that is compared to a control group receiving no treatment.

- IMPULS is deduced from experimental eye tracking research and integrates eye tracking as an objective measure to explore the efficacy of the intervention.

- IMPULS is not a multicentre trial, which might affect the generalisability of the findings. The group setting of the IMPULS trial, especially concerning food-related cue exposure, might affect the results compared to individual treatment.

- Concerning the compliance of study and treatment instructions, especially the homework between treatment sessions and overnight fasts before eye tracking, we have to rely on self-reports.

Trial registration number: German Clinical Trials Register, DRKS00007689, 14/01/2015, version from 11/06/2015, pre-results.

\section{INTRODUCTION}

Binge eating disorder

Binge eating disorder (BED) is an eating disorder that was approved for the first time in 
the new Diagnostic and Statistical Manual of Mental Disorders (DSM-5) 2013. ${ }^{1}$ It represents the most frequent eating disorder with a lifetime prevalence of $0.2-4.7 \%$ in the general population. ${ }^{2}$ BED is characterised by recurrent binge eating episodes and a concomitant subjective loss of control. Along with the binge eating, about $65 \%$ of patients with BED suffer from overweight or obesity leading to a twofold higher risk for obesity-associated somatic diseases. ${ }^{3}$

German treatment guidelines ${ }^{4}$ and practice guidelines of the American Psychiatric Association ${ }^{5}$ for eating disorders recommend psychotherapy as the treatment of first choice for BED with the reduction of binge eating episodes and overweight as the most important treatment goals. Cognitive-behavioural treatment (CBT) shows the most robust evidence of efficacy and high effect sizes concerning the reduction of binge eating episodes. ${ }^{6}{ }^{7}$ Outpatient CBT treatment of BED in individual versus group settings shows similar effects. ${ }^{4}{ }^{5}$ However, psychotherapeutic interventions for BED do not significantly reduce body weight and to date, it is unclear which elements of the used CBT interventions are effective. ${ }^{7}$ Therefore, the particular CBT interventions should be proved concerning their efficacy and there is a need for the advancement of treatment approaches for BED. ${ }^{6} 7$ One way to advance treatment is based on experimental research that explores potential underlying aetiological factors. One such promising aetiological factor in BED is impulsivity. ${ }^{8-10}$

\section{Impulsivity as a risk factor for BED}

Current experimental studies indicate that generally increased trait impulsivity ${ }^{8}$ and specifically increased impulsivity towards food and food cues ${ }^{9}$ represent risk factors for BED. Impulsivity is a multidimensional personality trait with a strong biological background ${ }^{11}$ that consists of two main factors: ${ }^{12}{ }^{13}$ (1) increased reward sensitivity or rather approach behaviour that means an increased purposive drive to reach hedonic stimuli and (2) rash-spontaneous behaviour or the decreased ability to resist this approach behaviour, that is, decreased inhibitory control.

One eye tracking study by our workgroup ${ }^{10}$ delivered evidence that people with BED differ from age-matched and weight-matched overweight/obese controls and agematched normal weight controls concerning an increased general and food-related impulsivity. People with BED pay more attention to food stimuli and rate those food stimuli as more rewarding than both control groups. Moreover, people with BED fail more often to inhibit eye movements towards food stimuli and to avert one's gaze especially from food stimuli. This suggests that people with BED perceive food as particularly rewarding and therefore develop increased craving towards food stimuli. Next, they begin to eat rashly and spontaneously without consideration of possible negative consequences like weight gain and are unable to inhibit or stop eating. ${ }^{9}{ }^{13}$ Hence, binge eating episodes represent a highly impulsive eating behaviour which is in line with the assumption that BED might represent a 'food addiction' disorder. ${ }^{14-16}$

\section{Impulsivity-focused treatment influencing BED pathology}

The few existing longitudinal studies concerning impulsivity and eating behaviour indicate that impulsivityfocused interventions are able to facilitate weight loss in people who diet ${ }^{17}$ and reduce self-selected meal sizes in non-clinical samples in the short term. ${ }^{18}{ }^{19}$ In obese samples, high impulsivity is associated with smaller weight reduction in a behavioural group treatment for children $^{20}$ and a weight loss programme for obese adults. ${ }^{21}$ Similarly, in inpatients with BED, high impulsivity is associated with less reduction of eating pathology in the long term. ${ }^{22}$ CBT in patients with eating disorders ${ }^{23}$ and CBT self-help or medical treatment in patients with $\mathrm{BED}^{24}$ seem to reduce impulsivity, especially reward sensitivity, along with eating disorder pathology. Moreover, pilot data from our workgroup show in a longitudinal eye tracking study in women with BED and overweight/obese controls that reductions in impulsivity after an outpatient CBT programme are associated with the reduction of binge eating frequency. Further, data from this same pilot study indicate that rash-spontaneous behaviour compared to reward sensitivity seems to be more easily alterable: Rash-spontaneous behaviour was reduced over time in both groups, the BED group that received CBT and the obese/overweight control group that did not receive CBT, whereas reward sensitivity did not change over time.

Taken together, current evidence indicates that eating behaviour and impulsivity interact and that impulsivity represents one risk factor for binge eating episodes in BED. ${ }^{22-24}$ Additionally, impulsivity seems to influence weight loss in people who diet and obese people. ${ }^{172021}$ Therefore, a group intervention that especially focuses on food-related impulsivity might increase self-control concerning impulsive eating behaviour. In that way, it might reduce binge eating episodes, food-related as well as general impulsivity, and possibly body weight in the long term. Pilot studies with overweight patients with bulimia nervosa, ${ }^{25}$ overweight children who eat in the absence of hunger, ${ }^{26}{ }^{27}$ and obese adults ${ }^{28}$ to whom selfregulation strategies and cue exposure in individual or group settings were provided strengthen this hypothesis: These impulsivity-focused interventions induced a significant reduction in binge eating frequency and body weight. However, studies that explore impulsivity-focused interventions in patients with BED are missing. Manualised therapy programmes in BED $^{29}{ }^{30}$ only marginally include impulsivity in their aetiological model of BED and use few interventions that refer to impulsive eating behaviour. Therefore, Gearhardt $e t a l^{16}$ recommend integrating interventions from addiction treatment, which focus on impulsivity and self-regulation. Similarly, Gerlach et $a l^{8}$ request strengthening of self-control skills in the treatment of obesity and related subgroups like in patients with BED. 
To the best of our knowledge, we are conducting the first randomised controlled trial to test the efficacy of a manualised group intervention that especially addresses food-related impulsivity in patients with BED. This study explores if an impulsivity-focused group intervention is able to reduce binge eating frequency, further eating pathology (eg, suffering from BED, grazing, irregular eating), food-related impulsivity as well as general impulsivity in the sense of a personality trait and body weight in the long term. We assess eating pathology by structured expert interviews and food-related as well as general impulsivity with the already used eye tracking paradigms ${ }^{10}$ and self-reports.

\section{STUDY AIMS AND HYPOTHESES}

The general aim of the IMPULS trial is to explore the efficacy of an impulsivity-focused group intervention in patients with BED (experimental group, EG) in comparison with a control group (CG), consisting of patients with BED who do not receive the group intervention. The group intervention will be provided as an outpatient group programme.

The following specific hypotheses are addressed:

- Hypotheses concerning the primary outcome: Significant reduction of the binge eating frequency over the past 4 weeks post-treatment versus pre-treatment and in the EG versus the CG

- Hypotheses concerning the secondary outcomes:

- Significant reduction post-treatment versus pretreatment and in the EG versus the CG concerning: (1) further eating pathology and (2) food-related and general impulsivity, that is, reward sensitivity and rash-spontaneous behaviour

- Significant reduction in the 3-month follow-up concerning: (1) binge eating frequency over the past 4 weeks, (2) further eating pathology, (3) body mass index (BMI in $\mathrm{kg} / \mathrm{m}^{2}$ ) and (4) general and food-related impulsivity.

Additionally, we explore binge eating episodes and other impulsive behaviours (eg, substance use, compulsive buying, irritability) in the past 7 days in process analyses. These process analyses are assessed weekly in an online questionnaire in the eight following weeks between pre-treatment and post-treatment.

In the EG, we assess the feasibility, acceptance and motivation concerning the impulsivity-focused group intervention at pre-treatment and post-treatment.

\section{METHODS AND ANALYSIS}

This study protocol has been written according to the SPIRIT statement (Standard Protocol Items for Randomised Trials). ${ }^{31}$

\section{Study design}

The IMPULS project is designed as a randomised controlled superiority trial with two parallel groups. Regarding the primary analysis, it has a $2 \times 2$ factorial design with the between-subjects factor 'group intervention' and the within-subjects factor 'measurement point'. Patients with BED participating in the group intervention (EG) are compared with patients with BED not participating in the group intervention (CG) at pre-treatment versus post-treatment ( $\mathrm{T} 0 \mathrm{vs} \mathrm{T} 1$ ). The primary hypothesis will be tested via the time versus treatment interaction term. Three months after termination of the group intervention in the EG and an equivalent time interval in the CG (T2), follow-up data will be assessed (secondary analysis, $2 \times 3$ factorial design). We compare changes concerning the primary and secondary end points in the EG versus CG from $\mathrm{T} 0$ to $\mathrm{T} 1$ and from $\mathrm{T} 1$ to $\mathrm{T} 2$.

\section{Participants}

The participants are randomised in the EG and CG after the first diagnostic appointment at T0. We decided to perform a stratified randomisation with the factors binge eating frequency over the past 4 weeks and BMI to balance the severity of BED and weight status as potential influencing factors. The data assessors inform the Institute for Clinical Epidemiology and Applied Biometry, Tübingen, Germany (ICEAB), which computes the stratification independently. Next, the ICEAB informs the principal investigator (PI) about group assignment.

Inclusion criteria in both groups comprise: (1) written informed consent, (2) age $\geq 18$ and (3) diagnosis of BED according to DSM-5. ${ }^{1}$ Exclusion criteria in both groups comprise: (1) current suicidality, (2) current bulimia nervosa, substance addiction, psychotic disorders, bipolar I disorder, (3) current psychotherapy, (4) pregnancy or lactation and (5) somatic diseases that influence eating behaviour or body weight (eg, diabetes, thyroid diseases) and in which medication has been adapted in the past 3 weeks.

\section{Sample size}

The sample size was calculated concerning the primary outcome, that is, the group (EG vs CG) xtime (T0 vs T1) interaction of the binge eating frequency over the past 4 weeks. In the EG, we estimated the magnitude of change in binge eating frequency based on longitudinal pilot data, which was $70 \%$. In the CG, we assumed a $30 \%$ reduction of binge eating frequency in terms of an estimated placebo effect. Hence, we aimed to recruit $\mathrm{n}=35$ people in each group to reach a power of $80 \%$ at the $\alpha$ level of 0.05 . Expecting a dropout rate of $10 \%$ as in other CBT programmes lasting 8 weeks, ${ }^{32}$ we decided to include 39 people in each group, resulting in 78 participants overall.

\section{Recruitment}

The IMPULS trial takes place at the Department of Psychosomatic Medicine and Psychotherapy, Tübingen, Germany. Participants are recruited via emails, bulletins, flyers, press releases and outpatient psychotherapists. People interested in study participation are screened for 
eligibility and receive verbal and written study information. If an individual meets the inclusion criteria, meets no exclusion criterion and gives written informed consent for study participation, he/she will be included into the trial. The first participant was enrolled in March 2015.

\section{Procedure}

The flow chart in figure 1 illustrates the study procedure. Each participant takes part at each of the three measurement points (T0, T1, T2). Each measurement point consists of two parts: first, the diagnostic assessment and second, the eye tracking assessment. Before the eye tracking assessment in the morning, each participant is instructed to fast overnight and receives a standardised breakfast directly before eye tracking.

After the first measurement point, the 8-week impulsivity-focused group intervention starts in the EG. The group intervention includes 8 weekly sessions in the evening, each taking $90 \mathrm{~min}$. Each group of the group intervention consists of five to six participants. In the same time period, both the EG and the CG participants perform the process analyses, that is, the weekly assessment of binge eating and other impulsive behaviour (duration 5 min per assessment).

After the group intervention, diagnostics and eye tracking measures are repeated directly afterwards (T1) and again 3 months later (T2). Data assessment and treatment are separated, that is, therapists of the group intervention are not aware about assessment outcomes, whereas data assessors are blind concerning the assignment to EG or CG.

\section{Outcome measures}

Data assessors are trained in diagnostic and eye tracking assessment and regularly supervised by the PI. The time schedule of the assessed data at the diagnostic and eye tracking assessments at each measurement point (T0, $\mathrm{T} 1, \mathrm{~T} 2$ ) is presented in table 1 . At $\mathrm{T} 1$ and $\mathrm{T} 2$, the diagnostic and eye tracking assessments are made directly one after the other and the diagnostic assessment is somewhat shortened in comparison with $\mathrm{T} 0$.

\section{Diagnostic assessment}

Body weight and height are measured in light clothing to compute BMI $\left(\mathrm{kg} / \mathrm{m}^{2}\right)$. The Eating Disorder Examination Interview (EDE; german version) ${ }^{33}$ is used to measure the primary outcome, that is, the binge eating frequency over the past 4 weeks, to verify the BED diagnosis and to exclude participants with other eating disorders. The Structured Clinical Interview for DSM-IV Disorders (SCID I german version) ${ }^{34}$ is used to assess other mental disorders. Several self-report measures (see table 1) are used to assess sociodemographic and clinical baseline data, eating behaviour, food-related and general impulsivity and depression levels.

\section{Eye tracking assessment of impulsivity}

The used eye tracking paradigms to measure food-related as well as general impulsivity are presented in figure 2 and slightly modified in comparison to Schag et al. ${ }^{10}$ Eye tracking is a non-invasive, objective and high-speed measurement to assess processes like impulse control. ${ }^{46} 47$ The two paradigms are presented in balanced order, so that half of the participants perform the cued exploration paradigm first and the other half perform the antisaccade paradigm first. In both paradigms, we use high-caloric food stimuli resembling typical binge food that are matched to neutral control stimuli concerning colour, contrast and luminance. These stimuli will be rated in the valence rating on computerised Likert scales: food stimuli concerning valence, palatability and craving and non-food stimuli concerning valence.

\section{Process analyses}

During the 8-week time interval of the group intervention, participants estimate binge eating and other impulsive behaviour weekly, that is, eight times overall, in an online questionnaire. ${ }^{48}$ By 'other impulsive behaviour', we mean specific behavioural expressions of impulsivity aside from binge eating like substance use, compulsive buying or irritability. We developed 10 categories of such other impulsive behaviours derived from the symptom criteria of impulse control disorders and borderline personality disorder according to ICD-10 (International Statistical Classification of Diseases and Related Health Problems) ${ }^{49}$ and DSM- $5^{1}$ and one category 'others' to cover impulsive behaviours extensively.

Participants have to report:

- The frequency of binge eating and other impulsive behaviour in the past 7 days

- The frequency of situations in which the participants have been able to inhibit binge eating or other impulsive behaviour

- Alternative behaviours shown instead of binge eating or other impulsive behaviour.

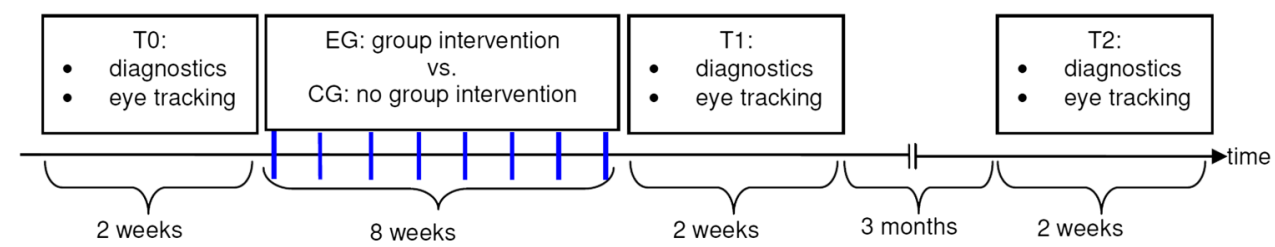

Figure 1 Study procedure. The weekly assessment points of the process analyses (assessment of binge eating and other impulsive behaviour) are marked with blue dashes. EG, experimental group; CG, control group. 
Table 1 Time schedule of each measurement point at T0, T1 and T2

\begin{tabular}{|c|c|}
\hline TO Diagnostic assessment & Eye tracking assessment \\
\hline $\begin{array}{l}\text { Body weight and height } \\
\text { - Sociodemographic and clinical } \\
\text { baseline data } \\
\text { Interviews: EDE, }{ }^{33} \text { SCID }{ }^{34} \\
\text { Questionnaires: EDE-Q, }{ }^{35} \text { DEBQ, }{ }^{36} \\
\text { FCQ-T-R, }{ }^{37} \text { BDI II }\end{array}$ & $\begin{array}{l}\text { Standardised breakfast } \\
\text { Eye tracking: antisaccade paradigm and free exploration paradigm in } \\
\text { balanced order with hunger and mood ratings directly before and after eye } \\
\text { tracking on visual analogue scales } \\
\text { Valence rating of presented food and non-food stimuli and FCQ-S } \\
\text { - Questionnaires: YFAS, }{ }^{40} \mathrm{BIS}-15,{ }^{41} \mathrm{BIS} / \mathrm{BAS}{ }^{42}\end{array}$ \\
\hline
\end{tabular}

T1 and T2: diagnostic and eye tracking assessment

- Body weight, height, clinical baseline data

- Standardised breakfast

- Eye tracking (s. T0)

- Valence rating of presented food and non-food stimuli and FCQ-S

- Interviews: EDE, SCID I (considering only the elapsed time until the last measurement)

- Questionnaires: EDE-Q, DEBQ, BDI II, BIS-15, BIS/BAS

BIS-15, Barratt Impulsiveness Scale short V; BIS/BAS, Behavioral Inhibition System/Behavioral Activation System questionnaire; BDI II, Beck Depression Inventory second V; DEBQ, Dutch Eating Behaviour Questionnaire; EDE, Eating Disorder Examination Interview; EDE-Q, Eating Disorder Examination Questionnaire; FCQ-S, Food Craving Questionnaire State; FCQ-T-R, Food Craving Questionnaire Trait short V; SCID I; Structured Clinical Interview for DSM-IV Disorders, Axis I; YFAS, Yale Food Addiction Scale.

\section{Adherence control}

In the EG and CG, attrition rate and study dropouts are assessed. In the EG, we additionally assess the frequency of attended sessions and treatment dropouts.

\section{Treatment evaluation}

In the EG, we assess feasibility, acceptance and motivation concerning the group intervention in evaluation questionnaires pre-treatment and post-treatment.

\section{Intervention}

We developed a treatment manual comprising structured CBT interventions focusing on the modification of food-related impulsivity. The group intervention targets both impulsivity components, increased reward sensitivity and rash-spontaneous behaviour. We mainly focus on rash-spontaneous behaviour, because this is the closest construct to binge eating behaviour and most likely to be malleable. Since pilot studies ${ }^{25-27}$ delivered evidence

A Cued exploration paradigm:

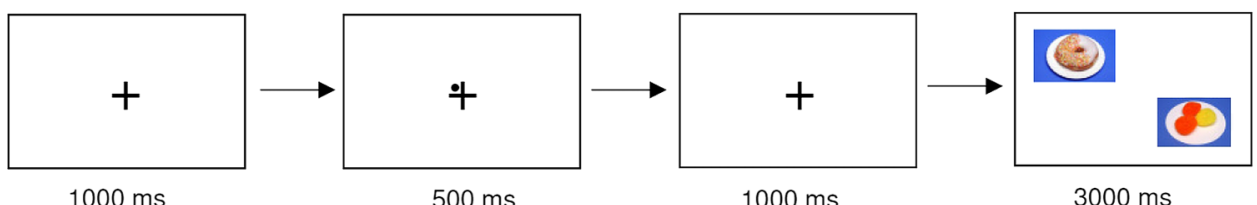

B Antisaccade paradigm:

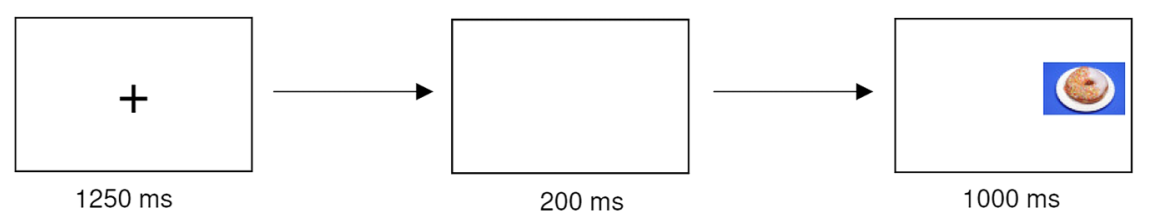

Figure 2 Schematic presentation of the cued exploration paradigm and the antisaccade paradigm.

(A) In the cued exploration paradigm, the participants are instructed to freely explore the food and non-food stimuli as if they were watching TV. Further, the participants are informed that a fixation cross and a dot are presented before in the middle of the screen, and that this dot is indicating the position of the next food stimulus. Afterwards, a fixation cross without a cue is displayed to ensure that no automatic orienting response is evoked. The dot, a so-called endogenous cue, enables the participant to direct attention voluntarily onto or away from the food stimulus. ${ }^{43} 44$ To assess reward sensitivity, we measure dwell time, fixation frequency and position of the first fixation on food versus non-food stimuli.

(B) In the antisaccade paradigm, the participants are instructed to look away in each trial from a stimulus that is appearing at the left or right site of the screen. Hence, the participant has to inhibit the automatic reaction towards newly appearing stimuli. Food and non-food stimuli are presented in randomised order. Before stimulus presentation, a fixation cross is presented to ensure that the participants have the same starting point at the middle of the screen that is followed by a so-called 'gap' to increase task difficulty. ${ }^{45}$ To assess rash-spontaneous behaviour and response inhibition, we measure first saccade errors (\%), second saccade errors (\%) and sequential errors, that is, subsequent errors of the first and second saccades. 
that food-related cue exposure as well as self-control strategies reduce binge eating behaviour and eating in the absence of hunger effectively, we especially focus on these two methods.

Table 2 gives an overview on the main topics of the single sessions. We use three psychotherapeutic methods in the group intervention: (1) psychoeducation, (2) selfmonitoring between sessions and development of adequate self-control strategies, and (3) food-related cue exposure with response prevention. The central concepts and methods have been adapted from structured CBT programmes, mainly from addiction therapy, ${ }^{50} 51$ approved BED manuals ${ }^{30}$ and handbooks on cue exposure in eating disorders and addictive disorders. ${ }^{52}$

According to our manual, treatment sessions are highly structured: Each session starts with a discussion about the self-monitoring homework and development of self-control strategies. In the main part, psychoeducation, joint or individual work, or food-related cue exposure with response prevention are conducted. To finish, the next session and homework are explained and prepared. Owing to the high structural degree and permanent self-monitoring between sessions, participants experience a more structured daily life, which addresses impulsivity aspects in general.

The group intervention is provided by trained and regularly supervised psychologists or physicians.

\section{Data analysis}

Data will be analysed with the Statistical Package of Social Sciences (SPSS). Owing to an expected dropout of $10 \%$, we perform intent-to-treat analyses with multiple imputations. Per protocol analyses are performed additionally. Concerning group and time differences and interactions, we use $2 \times 2$ factorial and in the follow-up $2 \times 3$ factorial repeated measure ANOVAs (analyses of variance) with the between-subjects factor 'group intervention' (EG, CG) and the within-subjects factor 'measurement point' (T0, T1, T2). Concerning eye tracking data, another within-subjects factor, the factor 'stimulus' (food, non-food), is given. Therefore, we perform $2 \times 2 \times 2$ or $2 \times 2 \times 3$ factorial repeated measure ANOVAs or compute so-called 'bias scores' representing differences in the performance of food versus non-food trials. If the requirements for ANOVAs concerning the primary outcome are violated, we test if the log-transformed primary outcome does fulfil the requirements. Otherwise and concerning other outcomes, we use nonparametric tests if the requirements for ANOVAs are violated.

Additionally, we compute correlational analyses exploratively to analyse relationships between outcome variables and influences of potential covariates like BMI, age or comorbidities. Regression analyses and analyses of covariance are used to explore influences of potential covariates concerning the primary outcome and eye tracking data. Further, we explore the data of the process analyses with mixed models and the evaluation questionnaires in the EG. Statistical significance is determined at $\alpha=0.05$.

\section{ETHICS AND DISSEMINATION \\ Data management and data monitoring}

All data are pseudonymised and will be stored at the Department of Psychosomatic Medicine and Psychotherapy for at least 10 years. Personal data of participants are saved in a separate password protected database with access only for the study staff. Source data in the IMPULS trial are defined as all data that are completed by the participants (eg, questionnaires), assessors (eg, interviews) or the PI (eg, (severe) adverse events) as well as all electronically assessed data (eg, eye tracking data, process analyses). The case report forms are the entered or imported data in SPSS. Only the study staff has access to the trial data set.

The Centre of Clinical Studies (CCS) at the University Hospital Tübingen verifies good clinical practice conformity and is responsible for regularly monitoring data. Two visits per year are planned as well as an initiation visit and a close-out visit. A monitoring report is written at each visit. The CCS monitors written informed consent and severe adverse events in all participants. In $25 \%$ of participants, inclusion and exclusion criteria, randomisation, adverse events, primary outcome, end of treatment and end of study are monitored. In one participant, source data and case report forms will be compared to $100 \%$.

We plan to publish the manual of the group intervention and study data in peer-reviewed journals.

Table 2 Structure of the group intervention in patients with binge eating disorder (BED)

\section{Main topics}

Initial phase (sessions 1, 2) Development of an impulsivity-based BED model

- Self-monitoring and development of individual treatment goals

Main phase (sessions 3-7) I Implementation of self-control strategies to reduce the probability of impulsive eating behaviour

- Development of new stimulus-response patterns and increasing self-efficacy by food-related cue exposure with response prevention

- Transfer to everyday life

Final phase (session 8) - Reflection, maintenance and transfer of achieved goals 


\section{Ethics and safety aspects}

This study has been approved by the ethics committee of the medical faculty of Eberhard Karls University Tübingen and the University Hospital Tübingen (471/2014BO2). All participants will be asked for written informed consent before inclusion into the study by the PI or data assessors. The IMPULS trial is registered at the German Clinical Trials Register (number: DRKS00007689).

Concerning patient safety, diagnostic and eye tracking assessments do not deliver specific health risks or side effects. All participants will be remunerated for their expenditure of time at the measurement points. Psychotherapeutic interventions might initially increase symptoms of mental disorders in the sense of raising consciousness of the problem. Thus, the group intervention might also increase BED symptoms or symptoms of other mental disorders in the short term. Since the impulsivity-focused group intervention does not replace standard treatment, participants are informed about further treatment possibilities. In the case of a severe somatic or psychiatric crisis like suicidality or severe complications, inpatient treatment will be provided to the participant and the study will be terminated for the participant.

\section{DISCUSSION}

The IMPULS study represents the first randomised controlled trial to test the efficacy of a manualised group intervention in patients with BED that especially addresses impulsivity. IMPULS explores impulsivity as one underlying aetiological factor of BED and integrates impulsivity into the theoretical framework of BED. Furthermore, IMPULS is deduced from experimental eye tracking research and integrates eye tracking to explore the efficacy of the group intervention. If IMPULS is able to reduce the binge eating pathology, we can conclude that interventions towards impulsivity represent one specific treatment factor of CBT for BED. Thus, the findings of the IMPULS project might clarify the role of impulsivity in CBT interventions and add to existing treatments by strengthening the implementation of self-control strategies and food-related cue exposure. Further, IMPULS elucidates the association between BED and addictive disorders and explores the possibility to transfer CBT interventions of addictive disorders on patients with BED.

After termination and analysis of the present pilot trial, we plan to conduct a multicentre randomised trial in order to further investigate efficacy of the present intervention. Eye tracking might especially represent one objective diagnostic tool concerning impulsive eating behaviour, rewarding sensitivity towards food and response inhibition failures. The decision to use a group setting in the IMPULS trial and especially to perform food-related cue exposure in a group is another important point that needs to be explored. If the group intervention is accepted by the participants and feasible in the group format, it might constitute an add-on group treatment to support individual psychotherapy.

Contributors KS, KEG, PM, WB and SZ participated in the design of the study. $\mathrm{KS}, \mathrm{EJL}, \mathrm{SB}$ and KEG developed the treatment manual. All the authors contributed to the study protocol. KS drafted the manuscript and all coauthors revised the manuscript critically and for important intellectual content. All the authors read and approved the final version of the manuscript.

Funding This work was supported by the Medical Faculty Tübingen within the framework of applied clinical science funding (grant number E.03.28024). The publication of this work was supported by the Deutsche

Forschungsgemeinschaft and Open Access Publishing Fund of University of Tübingen. KEG receives a grant from the Ministry of Science

Baden-Wuerttemberg and the European Social Fund and is partly supported by the Athene programme, a project of the Excellence Initiative of the German federal and state governments. These fundings had no role in the design of this study and will not have any role during its execution, analyses, interpretation of the data, or decision to submit results.

Competing interests None declared.

Patient consent Obtained.

Ethics approval Ethics committee of the Medical Faculty of Eberhard Karls University Tübingen and University Hospital Tübingen (471/2014B02).

Provenance and peer review Not commissioned; externally peer reviewed.

Open Access This is an Open Access article distributed in accordance with the Creative Commons Attribution Non Commercial (CC BY-NC 4.0) license, which permits others to distribute, remix, adapt, build upon this work noncommercially, and license their derivative works on different terms, provided the original work is properly cited and the use is non-commercial. See: http:// creativecommons.org/licenses/by-nc/4.0/

\section{REFERENCES}

1. American Psychiatric Association. Diagnostic and statistical manual of mental disorders-DSM-5. 5th edn. Arlington: American Psychiatric Publishing, 2013.

2. Kessler RC, Berglund PA, Chiu WT, et al. The prevalence and correlates of binge eating disorder in the world health organization world mental health surveys. Biol Psychiatry 2013;73:904-14.

3. Hudson JI, Hiripi E, Pope HG Jr, et al. The prevalence and correlates of eating disorders in the national comorbidity survey replication. Biol Psychiatry 2007;61:348-58.

4. Herpertz S, Herpertz-Dahlmann B, Fichter M, et al. S-3 Leitlinie Diagnostik und Behandlung der Essstörungen. Berlin: Springer, 2011.

5. Yager J, Devlin M, Halmi K, et al. Guideline watch (August 2012): practice guideline for the treatment of patients with eating disorders. 3rd edn. American Psychiatric Association, 2012.

6. Hay P. A systematic review of evidence for psychological treatments in eating disorders: 2005-2012. Int J Eat Disord 2013; 46:462-9.

7. Vocks S, Tuschen-Caffier B, Pietrowsky R, et al. Meta-analysis of the effectiveness of psychological and pharmacological treatments for binge eating disorder. Int J Eat Disord 2010;43:205-17.

8. Gerlach G, Herpertz S, Loeber S. Personality traits and obesity: a systematic review. Obes Rev 2015;16:32-63.

9. Schag K, Schönleber J, Teufel M, et al. Food-related impulsivity in obesity and binge eating disorder-a systematic review. Obes Rev 2013;14:477-95.

10. Schag K, Teufel M, Junne F, et al. Impulsivity in binge eating disorder: food cues elicit increased reward responses and disinhibition. PLOS ONE 2013;8:e76542.

11. Gray JA. The psychophysiological basis of introversion-extraversion Behav Res Ther 1970;8:249-66.

12. Gullo MJ, Loxton NJ, Dawe S. Impulsivity: four ways five factors are not basic to addiction. Addict Behav 2014;39:1547-56.

13. Dawe S, Loxton NJ. The role of impulsivity in the development of substance use and eating disorders. Neurosci Biobehav Rev 2004;28:343-51.

14. DiLeone RJ, Taylor JR, Picciotto MR. The drive to eat: comparisons and distinctions between mechanisms of food reward and drug addiction. Nat Neurosci 2012;15:1330-5. 
15. Smith DG, Robbins TW. The neurobiological underpinnings of obesity and binge eating: a rationale for adopting the food addiction model. Biol Psychiatry 2013;73:804-10.

16. Gearhardt AN, White MA, Potenza MN. Binge eating disorder and food addiction. Curr Drug Abuse Rev 2011;4:201-7.

17. Veling $\mathrm{H}$, van Koningsbruggen $\mathrm{GM}$, Aarts $\mathrm{H}$, et al. Targeting impulsive processes of eating behavior via the internet. Effects on body weight. Appetite 2014;78:102-9.

18. Houben $\mathrm{K}$, Jansen A. Training inhibitory control. A recipe for resisting sweet temptations. Appetite 2011;56:345-9.

19. van Koningsbruggen GM, Veling $\mathrm{H}$, Stroebe $\mathrm{W}$, et al. Comparing two psychological interventions in reducing impulsive processes of eating behaviour: effects on self-selected portion size. $\mathrm{Br} \mathrm{J}$ Health Psychol 2014;19:767-82.

20. Nederkoorn C, Jansen E, Mulkens S, et al. Impulsivity predicts treatment outcome in obese children. Behav Res Ther 2007:45:1071-5.

21. Murdaugh DL, Cox JE, Cook EW III, et al. fMRI reactivity to high-calorie food pictures predicts short- and long-term outcome in a weight-loss program. Neuroimage 2012;59:2709-21.

22. Fichter MM, Quadflieg N, Hedlund S. Long-term course of binge eating disorder and bulimia nervosa: relevance for nosology and diagnostic criteria. Int J Eat Disord 2008;41:577-86.

23. Shafran R, Lee M, Cooper Z, et al. Effect of psychological treatment on attentional bias in eating disorders. Int $J$ Eat Disord 2008:41:348-54.

24. Balodis I, Grilo C, Kober H, et al. A pilot study linking reduced fronto-striatal recruitment during reward processing to persistent bingeing following treatment for binge eating disorder. Int $J$ Eat Disord 2014;47:376-84.

25. Jansen A, Broekmate J, Heymans M. Cue-exposure vs self-control in the treatment of binge eating: a pilot study. Behav Res Ther 1992;30:235-41.

26. Boutelle KN, Zucker N, Peterson CB, et al. An intervention based on schachter's externality theory for overweight children: the regulation of cues pilot. J Pediatr Psychol 2014;39:405-17.

27. Boutelle KN, Zucker NL, Peterson CB, et al. Two novel treatments to reduce overeating in overweight children: a randomized controlled trial. J Consult Clin Psychol 2011;79:759-71.

28. McKee H, Ntoumanis N. Developing self-regulation for dietary temptations: intervention effects on physical, self-regulatory and psychological outcomes. J Behav Med 2014;37:1075-81.

29. Fairburn C. Cognitive behaviour therapy and eating disorders. New York/London: The Guilford Press, 2008.

30. Hilbert A, Tuschen-Caffier B. Essanfälle und Adipositas: Ein Manual zur kognitiv-behavioralen Therapie der Binge-Eating-Störung. Göttingen: Hogrefe, 2010.

31. Chan A-W, Tetzlaff J, Altman D, et al. SPIRIT 2013 statement: defining standard protocol items for clinical trials. Ann Intern Med 2013;158:200-7.

32. lacovino JM, Gredysa DM, Altman M, et al. Psychological treatments for binge eating disorder. Curr Psychiatry Rep 2012;14:432-46.

33. Hilbert A, Tuschen-Caffier B, Ohms M. Eating disorder examination: Deutschsprachige Version des strukturierten Essstörungsinterviews. Diagnostica 2004;50:98-106.

34. Wittchen $\mathrm{H}-\mathrm{U}$, Wunderlich U, Gruschwitz S, et al., eds. SKID I. Strukturiertes Klinisches Interview für DSM-IV. Achse I:
Psychische Störungen. Interviewheft und Beurteilungsheft. Eine deutschsprachige, erweiterte Bearbeitung der amerikanischen Originalversion des SCID I. Göttingen: Hogrefe, 1997.

35. Hilbert A, Tuschen-Caffier B, eds. Eating disorder examination questionnaire-Deutschsprachige Übersetzung. Münster: Verlag für Psychotherapie, 2006.

36. Grunert SC. Ein Inventar zur Erfassung von Selbstaussagen zum Ernährungsverhalten. Diagnostica 1989;35:167-79.

37. Meule A, Hermann T, Kübler A. A short version of the Food Cravings Questionnaire-Trait: the FCQ-T-reduced. Front Psychol 2014;5:190.

38. Hautzinger M, Keller F, Kühner C, eds. BDI-II. Beck Depressionsinventar, Revision - Manual. Frankfurt: Harcourt Test Services, 2006

39. Meule A, Lutz A, Vögele C, et al. Food cravings discriminate differentially between successful and unsuccessful dieters and non-dieters. Validation of the Food Cravings Questionnaires in German. Appetite 2012;58:88-97.

40. Meule A, Vögele C, Kübler A. Deutsche Übersetzung und Validierung der Yale Food Addiction Scale. Diagnostica 2012:58:115-26.

41. Meule A, Vögele C, Kübler A. Psychometrische Evaluation der deutschen Barratt Impulsiveness Scale-Kurzversion (BIS-15). Diagnostica 2011;57:126-33.

42. Strobel A, Beauducel A, Debener S, et al. Eine deutschsprachige Version des BIS/BAS-Fragebogens von Carver und White. Zeitschrift für Differentielle und Diagnostische Psychologie 2001;22:216-27.

43. Brignani D, Guzzon D, Marzi CA, et al. Attentional orienting induced by arrows and eye-gaze compared with an endogenous cue. Neuropsychologia 2009;47:370-81.

44. Mulckhuyse M, Theeuwes J. Unconscious attentional orienting to exogenous cues: A review of the literature. Acta Psychol (Amst) 2010;134:299-309.

45. Antoniades C, Ettinger U, Gaymard B, et al. An internationally standardised antisaccade protocol. Vision Res 2013;84:1-5.

46. Castellanos EH, Charboneau E, Dietrich MS, et al. Obese adults have visual attention bias for food cue images: evidence for altered reward system function. Int J Obesity 2009;33:1063-73.

47. Munoz DP, Everling S. Look away: the anti-saccade task and the voluntary control of eye movement. Nat Rev Neurosci 2004:5:218-28

48. UNIPARK. Online-Umfragen einfach erstellen. http://www.unipark. com/de/ (accessed 23 Jun 2015).

49. World Health Organization. International Statistical Classification of Diseases and Health Related Problems, Tenth Revision (ICD-10). German Modification, version 2013. http://www.dimdi.de/static/de/ klassi/icd-10-who/kodesuche/onlinefassungen/htmlamtl 2013/index. $\mathrm{htm}$ (accessed 1 Sep 2015).

50. Batra A, Bilke-Hentsch O. Praxisbuch Sucht: Therapie der Suchterkrankungen im Jugend- und Erwachsenenalter. Stuttgart: Thieme, 2012

51. Wölfling K, Jo C, Bengesser I, et al. Computerspiel- und Internetsucht: Ein kognitiv-behaviorales Behandlungsmanual. Stuttgart: Kohlhammer, 2013.

52. Neudeck P, Wittchen H-U. Konfrontationstherapie bei psychischen Störungen. Göttingen: Hogrefe, 2005. 